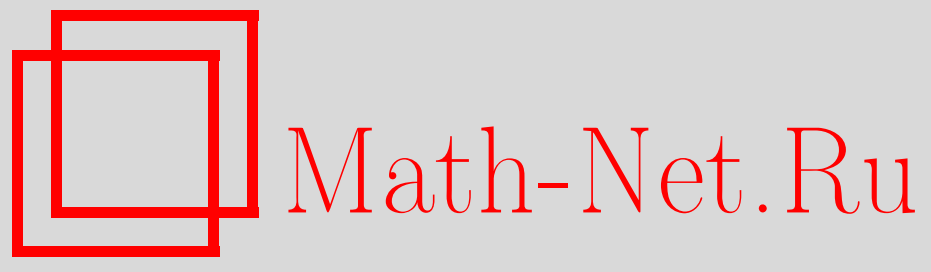

С. Ю. Немировский, Р. Г. Шафиков, Гипотезы Чена и Рамаданова, УМH, 2006, том 61, выпуск 4, 193-194

DOI: https://doi.org/10.4213/rm2614

Использование Общероссийского математического портала Math-Net.Ru подразумевает, что вы прочитали и согласны с пользовательским соглашением http://www . mathnet.ru/rus/agreement

Параметры загрузки:

IP: 54.237 .206 .68

26 апреля 2023 г., $15: 16: 46$

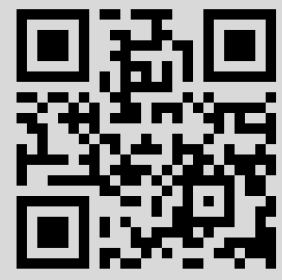




\section{Гипотезы Чена и Рамаданова}

\section{С. Ю. Немировский, Р. Г. Шафиков}

В этой заметке мы применяем теорему об униформизации из наших работ [1], [2] для обобщения результата Фу и Вонга [3] о связи двух известных гипотез о поведении метрики Бергмана строго псевдовыпуклой области в $\mathbb{C}^{n}, n \geqslant 2$.

1. Пусть $D \Subset \mathbb{C}^{n}$ - произвольная ограниченная область. Кернфункиия Бергмана области $D$ может быть определена формулой $K_{D}(z):=\sum_{j=1}^{\infty} \varphi_{j}(z) \overline{\varphi_{j}(z)}$, где $\left\{\varphi_{j}\right\}_{j=1, \ldots, \infty}-$ любой ортонормированный базис гильбертова пространства $L^{2} \mathscr{O}(D)$ голоморфных функций с интегрируемым квадратом в области $D$.

Хорошо известно, что функция $\log K_{D}(z)$ строго плюрисубгармонична, а замкнутая положительная $(1,1)$-форма $k_{D}:=i \partial \bar{\partial} \log K_{D}(z)$ инвариантна относительно биголоморфных отображений между ограниченными областями. Метрикой Бергмана области $D$ называется кэлерова метрика, соответствующая этой кэлеровой форме.

2. Классическая задача, ставившаяся в разной форме Бергманом, Хуа и Яу, состоит в описании области в терминах дифференциально-геометрических свойств ее метрики Бергмана. Так, ограниченная область с полной метрикой Бергмана постоянной голоморфной секционной кривизны биголоморфна шару по известной теореме Лю Чи-кена [4].

Гипотеза Чена [5] утверждает, что для строго псевдовыпуклой области с гладкой границей сделанные в теореме Лю предположения могуть быть существенно ослаблены. А именно, такая область биголоморфна шару тогда и только тогда, когда ее метрика Бергмана является метрикой Кэлера-Эйнштейна. (Формулировка этой гипотезы в заметке [5] несколько расплывчата. Точное утверждение выше взято из работы [3].)

3. Фефферман [6] (см. также [7]) установил следующий глубокий результат о граничном поведении кернфункции строго псевдовыпуклой области $D$ с гладкой границей. Пусть $\rho \in C^{\infty}(\bar{D})$ - это некоторая определяющая функция области $D$. Тогда существует представление

$$
K_{D}(z)=\varphi(z) \rho(z)^{-(n+1)}+\psi(z) \log |\rho(z)|,
$$

где функции $\varphi, \psi \in C^{\infty}(\bar{D})$, причем $\varphi$ не обращается в нуль нигде на $\partial D$. Заметим, что последнее свойство влечет за собой полноту метрики Бергмана строго псевдовыпуклой области.

Несмотря на то, что кернфункция определяется глобально, ее асимптотическое поведение при $z \rightarrow z_{0} \in \partial D$ зависит только от локальной CR-геометрии границы в точке $z_{0}$ (см. [6]). Например, для кернфункции единичного шара $B \subset \mathbb{C}^{n}$ имеется явное выражение $K_{B}(z)=\frac{n !}{\pi^{n}}\left(1-\|z\|^{2}\right)^{-(n+1)}$ с тождественно нулевым логарифмическим членом. Таким образом, если граница строго псевдовыпуклой области $D$ сферична (т.е. локально CR-диффеоморфна единичной сфере $\partial B \subset \mathbb{C}^{n}$ в каждой своей точке $q \in \partial D)$, то коэффициент $\psi$ при логарифмическом члене в представлении (1) обращается в нуль с бесконечным порядком на границе этой области.

Гипотеза Рамаданова [8] утверждает, что и обратно, из условия $\psi(z)=O\left(\rho^{\infty}\right)$ при $z \rightarrow \partial D$ следует сферичность границы области $D$. Для областей в $\mathbb{C}^{2}$ эта гипотеза была доказана Грэмом и Бернсом (см. [9]) и Буте де Монвелем [10].

Работа первого автора поддержана Российским фондом фундаментальных исследований (грант № 05-01-00981) и программой "Современные проблемы теоретической математики" ОМН РАН. Второй автор был поддержан грантом Natural Sciences and Engineering Research Council of Canada. 
4. Теперь мы можем сформулировать и доказать основные результаты работы.

Теорема. Гипотеза Чена в $\mathbb{C}^{n}$ следует из гипотезы Рамаданова в $\mathbb{C}^{n}$.

Поскольку гипотеза Рамаданова доказана в $\mathbb{C}^{2}$, мы получаем, что гипотеза Чена также верна в $\mathbb{C}^{2}$, т.е. имеет место следующее утверждение.

Следствие. Метрика Бергмана строго псевдовыпуклой области $D \Subset \mathbb{C}^{2}$ с гладкой границей является метрикой Кэлера-Эйнштейна тогда и только тогда, когда эта область биголоморфна шару.

ЗАмечАниЕ. Фу и Вонг [3] доказали эти результаты в частном случае односвязных областей, используя более слабую теорему об униформизации Черна и Жи [11], и сформулировали общий случай в качестве открытого вопроса.

ДокАЗАТЕЛЬСтво теоремы. Предположим, что метрика Бергмана строго псевдовыпуклой области $D$ является метрикой Кэлера-Эйнштейна. Фу и Вонг [3] вычислили (весьма остроумно), что в этом случае коэффициент $\psi$ при логарифмическом члене в разложении (1) обращается в нуль с бесконечным порядком на границе области. Приняв гипотезу Рамаданова, мы получаем, что граница области $D$ сферична. Следовательно, область $D$ накрывается единичным шаром по теореме об униформизации (см. теорему А.2 в [1] и следствие 3.2 в [2]).

Поскольку область $D$ является фактором шара по группе голоморфных преобразований скольжения, на ней существует естественная полная метрика постоянной голоморфной секционной кривизны, которая получается как фактор стандартной инвариантной метрики в шаре. Согласно Чену и Яу [12], полная метрика Кэлера-Эйнштейна на $D$ единственна с точностью до постоянного множителя. Поэтому метрика Бергмана области $D$ пропорциональна факторметрике, а значит, имеет постоянную голоморфную секционную кривизну. Отсюда по теореме Лю [4] вытекает, что область $D$ биголоморфна шару.

Пример. Область $D_{B S}=\left\{\left.(z, w) \in \mathbb{C}^{2}|\sin \log | z|+| w\right|^{2}<0, e^{-\pi}<|z|<1\right\}$, построенная Бернсом и Шнайдером [13], является примером неодносвязной строго псевдовыпуклой области со сферической границей в $\mathbb{C}^{2}$. Таким образом, кернфункция этой области не имеет логарифмической асимптотики на границе, но ее метрика Бергмана не является метрикой Кэлера-Эйнштейна.

\section{Список литературы}

[1] С. Ю. Немировский, Р. Г. Шафиков, Изв. РАН. Сер. матем., 69:6 (2005), 115-130. [2] С. Ю. Немировский, Р. Г. Шафиков, Изв. РАН. Сер. матем., 69:6 (2005), 131-138. [3] S. Fu, B. Wong, Math. Res. Lett., 4:5 (1997), 697-703. [4] Q.-K. Lu, Acta Math. Sinica, 16, 269-281; Chinese Math.-Acta, 8 (1966), 283-298. [5] S.-Y. Cheng, Conference on non-linear problems in geometry (Katata, September 3-8, 1979), Tohoku University, Department of Mathematics, Sendai, 1979, 2. [6] C. Fefferman, Invent. Math., 26 (1974), 1-65. [7] L. Boutet de Monvel, J. Sjöstrand, Journées: Équations aux Dérivées Partielles de Rennes (1975), Asterisque, 34-35, Soc. Math. France, Paris, 1976, 123-164. [8] I. P. Ramadanov, C. R. Acad. Bulgare Sci., 34:7 (1981), 927-929. [9] C. R. Graham, Complex analysis, II (College Park, MD, 1985-86), Lecture Notes in Math., 1276, Springer, Berlin, 1987, 108-135. [10] L. Boutet de Monvel, The Bergman kernel in dimension 2, Séminaire sur les Équations aux Dérivées Partielles 1987-1988, exp. 22, École Polytech., Palaiseau, 1988. [11] S. S. Chern, S. Ji, Ann. of Math. (2), 144:2 (1996), 421-439. [12] S.-Y. Cheng, S.-T. Yau, Comm. Pure Appl. Math., 33:4 (1980), 507-544. [13] D. Burns, Jr., S. Shnider, Invent. Math., 33:3 (1976), 223-246.

\section{C. Ю. Немировский (S. Yu. Nemirovskii)}

Математический институт им. В. А. Стеклова РАН;

Ruhr-Universität Bochum, Germany

E-mail: stefan@mi.ras.ru

\section{P. Г. Шафиков (R. G. Shafikov)}

University of Western Ontario, London, Canada

E-mail: shafikov@uwo.ca
Представлено А. Г. Сергеевым Принято редколлегией 14.06.2006 